\title{
TAXA DE DESEMPREGO E A ESCOLARIDADE DOS DESEMPREGADOS NOS ESTADOS BRASILEIROS: ESTIMATIVAS DINÂMICAS DE DADOS EM PAINÉIS*
}

\author{
Gilberto J. Fraga ${ }^{\S}$ \\ Joilson Dias ${ }^{\text {a }}$
}

\begin{abstract}
RESUMO
O objetivo deste trabalho é estender o modelo teórico de desemprego especificado por Marston (1985) e aplicálo para os Estados brasileiros, com destaque especial na avaliação do papel exercido pela escolaridade (capital humano) dos desempregados na taxa de desemprego. Nesta extensão do modelo, também são considerados como fatores atrativos da localidade a diferença entre o salário médio local e o nacional e a estrutura produtiva da economia. Na parte econométrica, a principal inovação está nas estimativas dinâmicas em painéis de dados, que elimina o problema de variáveis omitidas e de endogeneidade, comum nestes casos. Como resultado principal, constatou-se que a escolaridade dos desempregados possui um efeito não-linear. Somente para níveis de escolaridade média dos desempregados acima de quatro anos, o efeito sobre a taxa de desemprego é negativo, ou seja, contribui para diminuir a taxa de desemprego. Com relação à estrutura da economia, o setor agrícola demonstrou ser o único que reduz a taxa de desemprego, uma vez considerada a escolaridade.
\end{abstract}

Palavras-chave: desemprego, escolaridade, efeitos fixos, estimativas dinâmicas.

\begin{abstract}
The objective of this paper is to extend the unemployment theoretical model developed by Marston (1985) and apply it to the Brazilian States data. The main aspect of the proposed extension regards to the role exerted by the unemployed average human capital. As local attractive, the extended model considers the role of the average wage difference from the national and the economy structure. On the econometrics side, the main contribution regards to the dynamic panel data estimates that takes into consideration the endogeneity and omitted variables problems. The results are that the unemployed level of the education has a non-linear effect on the unemployment rate. For average levels of education above four years the education level led the unemployment rate to diminish. The state economy sector that helps to decrease the unemployment rate was the agricultural one, once the educations was accounted for.
\end{abstract}

Key words: unemployment, education; fixed-effect, dynamic estimates.

JEL classification: C33, C52, E24, J64.

\footnotetext{
* Os autores gostariam de agradecer as contribuições feitas por dois pareceristas anônimos e, ao mesmo tempo, isentá-los da responsabilidade por eventuais erros remanescentes.

§ Programa de Pós-Graduação em Economia - UEM. E-mail: gjfraga@yahoo.com.br

a Professor Titular do Departamento de Economia da Universidade Estadual de Maringá - UEM. Endereço para contato: Av. Colombo, 5790 - Bloco: C23 - Sala 10 - Maringá - PR CEP 87020-900. E-mail: jdias@uem.br. O autor agradece o apoio recebido do CNPq.

Recebido em janeiro de 2007. Aceito para publicação em setembro de 2007.
} 


\section{INTRODUÇÃo}

A taxa de desemprego no Brasil, no ano de 2003, foi substancialmente superior à taxa registrada no ano de 1995, algo em torno de 1,5 vezes. ${ }^{1}$ Este aumento do desemprego após a estabilização econômica passou a ser um dos alvos a serem minimizados pela política econômica de longo prazo. Atualmente, as recomendações para diminuir a taxa de desemprego ${ }^{2}$ têm concentrado boa parte da sua atenção em políticas de qualificação do capital humano. Os estudos da importância das políticas de investimento em qualificação sendo realizadas na economia brasileira em período recente podem ser encontrados nos trabalhos de Gonzaga et al. (2002); Chahad (2003); Reis (2004) e Giovannetti e Menezes-Filho (2005).

Nesta perspectiva, este artigo preocupa-se com a importância da acumulação do capital humano de longo prazo e não somente com a qualificação do mesmo. Portanto, busca-se medir o papel exercido pelo capital humano médio como forma de aumentar a possibilidade de emprego dos indivíduos, refletindo os desenvolvimentos teóricos ocorridos recentemente na teoria do capital humano.

Entretanto, diante das características específicas das economias dos Estados brasileiros, este efeito de melhor escolaridade média pode ser exacerbado ou reduzido sobre a empregabilidade dos indivíduos desempregados. A razão seria a heterogeneidade da economia brasileira em vários aspectos como econômico e geográfico, tornando-se, por vezes, inviáveis análises agregadas de variáveis como desemprego. Assim, para se obter um resultado mais acurado, necessita-se de uma estratificação maior, pois, em análises agregadas da taxa de desemprego, desconsideram-se diferenças regionais marcantes. Segundo Courseuil et al. (1999), se o desemprego for um fenômeno endêmico de uma região do País apenas, e não um fenômeno nacional, as políticas particulares de combate ao desemprego deveriam ter um caráter regional, pois políticas nacionais poderiam gerar resultados ineficientes. Esta ineficiência das políticas pode ocorrer especialmente quando as taxas de desemprego divergem dentro e entre as economias das regiões ou Estados de um país. Para compreender o quanto estes diferenciais são permanentes e/ou dinâmicos no tempo, surgiu a teoria compensatória de Marston (1985). Em síntese, esta teoria busca explicar porque as taxas de desemprego possuem um diferencial permanente entre as regiões. ${ }^{4}$

Neste cenário, o objetivo deste trabalho é estender o modelo de Marston (1985) e aplicá-lo aos Estados brasileiros, considerando o impacto de alguns aspectos dos Estados na taxa de desemprego. Esta modificação no modelo será por meio da incorporação do nível médio de escolaridade dos desempregados. A razão está em que a teoria compensatória, apesar de sugerir a importância das diferenças em atratividades das regiões ou Estados, desconsidera esse importante elemento que mede uma das características relevantes dos desempregados. E esta omissão pode introduzir o problema de viés nas estimativas.

Este aspecto torna-se ainda mais importante, pois muda o conceito de política econômica a ser seguida. Por exemplo, se a escolaridade contribuir para reduzir a taxa de desemprego dos Estados conforme se espera que ocorra, o foco das políticas para com o grupo de desempregados deveria enfatizar esta melhoria de conhecimento como objetivo primordial e com enfoques diferentes para

1 Estatísticas obtidas a partir dos dados da Pesquisa Nacional por Amostra de Domicílios (PNAD).

2 No presente trabalho, um indivíduo (trabalhador) é considerado desempregado se ele tem entre 10 e 65 anos de idade, não trabalhou nos últimos 30 dias e está procurando emprego.

3 Veja Kruger e Lindahl (1999), por exemplo.

4 Na Seção 2 será feita uma apresentação adequada da teoria e do modelo proposto pelo autor. 
cada Estado ou região. Os demais aspectos específicos dos Estados a serem considerados, são: i) a estrutura produtiva (PIB setorial) que contempla a heterogeneidade da demanda por trabalhadores, sendo que em alguns Estados (regiões) concentram-se forte setor de serviços e indústria, enquanto outros são mais voltados para agricultura; ii) os salários médios reais/hora para verificar o diferencial de salários de cada Estado em relação ao nível nacional, e iii) a variável índice de Gini que capta as distorções presentes na distribuição de renda em determinada região, podendo influenciar na atratividade da mesma. Estas variáveis compõem o que se denomina, no modelo, de atratividade da região.

Outra particularidade do artigo está relacionada com a metodologia de estimação. A questão de variáveis omitidas deve ser sempre considerada uma vez que uma parcela da diferença entre os Estados está relacionada a aspectos fixos como geografia, cultura e políticas públicas, que normalmente não são capturados em estimativas. Além disto, uma parcela destes aspectos pode ter comportamentos dinâmicos. Assim, o modelo a ser estimado em painel de dados considera os aspectos dinâmicos desta relação, bem como os aspectos de endogeneidade e omissão de variáveis.

Quanto às variáveis que representam a atratividade e, portanto, diferenciam os Estados, pressupõem-se as seguintes relações: i) espera-se que os Estados que tiverem salários ${ }^{5}$ superiores à média nacional possuam maior taxa de desemprego, pois geram maior atratividade. Desta forma, o salário médio/hora, considerado uma variável-chave na determinação do desemprego de cada Estado, será representado pelo diferencial em relação à média nacional; ii) o coeficiente Gini (geral) apresenta a estrutura da distribuição de renda dos Estados. Se o seu efeito for negativo significa que, quanto menos eqüitativa for a distribuição do rendimento do trabalho, menor será a taxa de desemprego; iii) a estrutura da economia representa o diferencial entre os setores econômicos dos Estados. Espera-se que economias com um setor de serviços predominante atraiam uma quantidade maior de trabalhadores com nível de conhecimento acima da média, enquanto que, com o agrícola, espera-se exercer efeito oposto. Neste caso, os sinais destes setores, se negativos, indicam que as políticas que fomentam determinado setor são benéficas à redução da taxa de desemprego dos Estados. Por exemplo, uma política de fomento ao setor agrícola pode resultar em aumento na oferta de empregos dos Estados cujo grau de escolaridade média esteja abaixo da nacional.

Sumariamente, as contribuições deste trabalho são de duas naturezas. A primeira é sobre o objetivo de verificar a contribuição direta dos conhecimentos dos desempregados na taxa de desemprego dos Estados, uma vez consideradas suas características específicas. A segunda está associada à questão da metodologia econométrica, que utiliza o sistema de equações dinâmicas, sugerido por Arellano e Bond (1991). ${ }^{6}$

Este trabalho está organizado da seguinte forma: Seção 1, Introdução; Seção 2, apresentação da teoria compensatória de Marston e a nova especificação do modelo; Seção 3, apresentação das estimativas econométricas, nas quais primeiramente estimamos dois modelos: efeitos fixos e randômicos. A seguir, estimamos o modelo dinâmico e, por fỉm, são apresentadas as considerações finais.

5 Taylor (1975) apresenta modelo(s) sobre a determinação dos salários.

6 Esta metodologia, sugerida por Arellano e Bond (1991), continuou sendo complementada pelos trabalhos de Ahn e Schmidt (1995), Arellano e Bover (1995), Blundell e Bond (1998). 


\section{TEORIA COMPENSATÓRIA}

O objetivo desta seção é apresentar e estender a teoria compensatória desenvolvida por Marston (1985). Essa teoria parte do pressuposto de que existe um equilíbrio entre salário, atratividade e desemprego de uma determinada região. Este equilíbrio seria determinado da seguinte forma: quanto maior o salário, ou mais atrativa fosse uma região (Estado) em relação às demais, mais trabalhadores seriam atraídos para aquela região e, portanto, maior seria a taxa de desemprego.

Estes argumentos estão formalizados em um modelo que leva em consideração estas variáveis, que, por sua vez, afetam o nível de utilidade alcançado pelos trabalhadores. Considera-se a hipótese geral de que os indivíduos de uma economia atribuem um mesmo nível de satisfação a uma cesta de consumo (C) e às atratividades mencionadas (A). Os indivíduos são limitados pela sua renda do trabalho, a qual é condicionada pela probabilidade de estar desempregado (U). Como resultado, tem-se a seguinte função de maximização da utilidade dos trabalhadores (u):

$$
\begin{aligned}
& \max u(\underset{+}{C, A}) \text { desde que W.f }[(1-\mathrm{U}), \mathrm{g}(\mathrm{E}, \mathrm{S})]=\mathrm{C} . \\
& \text { sendo, } u(C)>0 \text {; e } u^{\prime \prime}(C)<0
\end{aligned}
$$

onde, $W=\left(\overline{W_{0}}+W_{e}\right)$ representa o salário total, $\overline{W_{0}}$ o salário médio nacional, $W_{e}$ é o diferencial de salário devido a características da localidade ou do indivíduo, $A$ é a atratividade da região, $U$ é a taxa de desemprego ou a probabilidade média de o indivíduo ficar desempregado, $E$ é o nível de escolaridade dos desempregados em determinada região e $S$ é o PIB setorial. W.f $f(1-U), g(E$, $S)$ ] representa a renda esperada pelo trabalhador em determinada região. As variáveis $W_{0}, U$ e $A$ são consideradas como dadas para o trabalhador. Então, a função de utilidade indireta dos trabalhadores de uma região pode ser representada da seguinte forma:

$$
\begin{aligned}
& V(W, U, A, E, S)=\max _{C, \lambda}\{u(C, A)+\lambda[W \cdot f[(1-U), g(E, S)]-C]\} \\
& V_{w}>0, V_{u}<0, V_{a}>0, V_{E}>0, V_{S}>0 .
\end{aligned}
$$

Sendo que o termo da equação multiplicado por $\lambda$ representa a restrição orçamentária do trabalhador.

O resultado do modelo é que, em equilíbrio, o nível de utilidade alcançado pelos trabalhadores deve ser o mesmo em todas as regiões.

$$
V\left[\left(W_{0}+W_{e}\right)^{*}, U^{*}, A, E, S\right]=K
$$

onde $K$ é uma constante. ${ }^{7}$ Observa-se que a igualdade entre as taxas de desemprego não é uma condição necessária para a igualdade do nível de utilidade indireta. Desta forma, um diferencial persistente entre as taxas regionais de desemprego é um resultado que poderá acontecer nesse modelo. Esse diferencial persistente pode ser explicado, por exemplo, por uma diferença de atratividade das regiões. Diferenciando (3):

$7 \quad \mathrm{O}\left({ }^{*}\right)$ indica o valor de equilíbrio da variável correspondente. 


$$
\begin{aligned}
& V_{w} d\left(W+W_{e}\right)^{*}+V_{u} d U^{*}+V_{a} d A+V_{E} d E+V_{s} d S=0 \\
& d U^{*}=-\left|\frac{V_{a}}{V_{u}}\right| d A-\left|\frac{V_{w}}{V_{u}}\right|\left(d \bar{W}+d W_{e}\right)-\left|\frac{V_{E}}{V_{u}}\right| d E-\left|\frac{V_{s}}{V_{u}}\right| d S \\
& d U^{*}=U_{t}-U_{t-1} ; d A=A_{t}-A_{t-1} ; d W=W_{t}-W_{t-1} ; d E=E_{t}-E_{t-1} ; d S=S_{t}-S_{t-1}
\end{aligned}
$$

Portanto, a taxa de desemprego de equilíbrio pode ser escrita da seguinte forma:

$$
U_{t}^{*}=U_{t-1}+a d A+\varphi d \bar{W}+\varphi d W_{e}+a d E+a d S
$$

De acordo com a equação (4), alterações em um dos argumentos da função $V$ devem ser compensados por alterações em pelo menos um dos outros argumentos da função, se houver variações nos salários ou nas atratividades e se estas alterações puderem ser compensadas por variações na taxa de desemprego para restabelecer a igualdade da utilidade entre as regiões.

A equação (5) apresenta a forma da equação estrutural a ser estimada, visando estabelecer o equilíbrio da taxa de desemprego entre as regiões. Portanto, dependendo da alteração inicial ter sido observada no salário $W=\left(\overline{W_{0}}+W_{e}\right)$, atratividades $(A)$, nível de conhecimento acumulado (E) e/ou estrutura produtiva (S), o sistema ajusta de forma dinâmica para compensar estas oscilações no longo prazo. Uma forma reduzida do ponto de vista econométrico da equação acima é a seguinte:

$$
U_{i t}^{*}=U_{i, t-1}^{*}+\zeta_{i t}
$$

Esta equação (6) separa o desemprego de equilíbrio de outros componentes (variáveis) que afetam a taxa de desemprego da área. Mais especificamente, $\zeta_{i t}$ representa as componentes observáveis e não-observáveis do modelo econométrico a ser estimado.

Marston, com base no censo americano de 1970, mostra evidências que corroboram a teoria. Pissarides e McMaster (1990), com dados do Reino Unido, também aplicaram a teoria e verificaram que, no longo prazo, a economia converge para o equilíbrio compensatório.

\subsection{Especificação empírica do modelo estendido da teoria compensatória}

A especificação empírica do modelo da seção anterior propõe um modelo geral para taxa de desemprego dos Estados brasileiros, que agrega, além dos efeitos específicos dos Estados, também o efeito direto relacionado aos desempregados, ou seja, a escolaridade média. Assim, o modelo geral a ser estimado possui os seguintes componentes:

$$
U_{i t}=\beta U_{i, t-1}+\varphi d W_{i, t}+\gamma d A_{i, t}+\delta d E_{i, t}+\tau d E_{i, t}^{2}+\phi d S_{i, t}+\left(\alpha_{i}+\eta_{i, t}\right)
$$

onde os componentes observáveis são: $W_{i, t}$ é o diferencial de salários médios dos Estados (regiões), $A_{i, t}$ é uma matriz $n \times k$ observações que representam a variável Gini e $S$ participação do PIB dos 
setores, um bom desempenho da variável gini (menor desigualdade) torna a região em consideração relativamente mais atrativa. $E_{i, t}$ é a escolaridade média dos desempregados; e os não-observáveis são: $\alpha_{i}$ representa os efeitos fixos não-observáveis e $\eta_{i, t}$ representa choques e alterações dinâmicas no tempo.

A inclusão do $E$ (nível da escolaridade média dos desempregados) e seu valor ao quadrado $\left(E^{2}\right)$ está baseado no trabalho de Dias et al. (2005). A inclusão da variável escolaridade de forma não-linear visa captar, especificamente, os diferentes níveis de capital humano acumulado pelos trabalhadores desempregados ao longo do tempo. Portanto, mede de forma indireta a importância de políticas educacionais dos Estados. Logo, estimando a equação (7), pode-se calcular a importância das variáveis de interesse, dos efeitos observáveis e outros não-observáveis quantificados pelas estimações.

\subsection{Aplicações econométricas}

Os dados utilizados neste trabalho referem-se aos 26 Estados brasileiros mais o Distrito Federal no período de 1995-2003, ${ }^{8}$ contemplando um total de 243 observações disponíveis. As variáveis 1, 8 e 9, listadas abaixo, são o conjunto de dados da PNAD $^{9}$ (Pesquisa Nacional por Amostra de Domicílios) para indivíduos entre 10 e 65 anos de idade residentes nas áreas urbanas. As demais variáveis foram obtidas no IPEADATA ${ }^{10}$ (Instituto de Pesquisa Econômica Aplicada). As descrições das variáveis utilizadas são:

1) taxa de desemprego do Estado, txdes;

2) Índice de Gini (geral) do Estado, gini;

3) média do salário hora $(\mathrm{R} \$ / \mathrm{Hrs})$ de cada Estado dividida pela média nacional forma o índice de atratividade do salário do Estado, w;

4) participação do setor serviços no PIB do Estado, pibserv;

5) participação do setor industrial no PIB do Estado, pibind;

6) participação do setor agropecuário no PIB do Estado, pibagro;

7) participação do setor comércio no PIB do Estado, pibcome;

8) escolaridade média dos desempregados do Estado, $E$;

9) escolaridade média ao quadrado dos desempregados do Estado, $E^{2}$.

Na tabela a seguir, a primeira variável refere-se à taxa de desemprego (txdes), cuja média no período compreendido foi de $10,27 \%$, com um desvio padrão de 2,66\%. Os valores máximos de 24,56\% e mínimos de 4,26\% refletem existência de discrepâncias nos dados, que é prevista. Estas taxas provavelmente apresentam-se como potenciais outliers. Durante o processo de estimação, estas observações serão minimizadas, em especial durante a utilização do método dinâmico de estimação. A média dos salários dos Estados está muito próxima da média do salário nacional no período em consideração, pois representa 98,97\% da média do salário nacional; sendo que o menor salário médio observado representou 35,9\% da média nacional e o maior salário médio observado foi $146 \%$ superior ao valor da média do salário nacional. A leitura das estatísticas das demais variáveis obedece ao mesmo formato das já relatadas.

8 O período dos dados encerra em 2003, porque, na realização da pesquisa, não havia disponibilidade das PNAD's posteriores; no entanto, entende-se que tal fato não compromete as conclusões do trabalho.

9 No ano de 2000, não houve Pesquisa Nacional por Amostra de Domicílios, portanto, foi elaborada uma média para esse ano.

10 www.ipeadata.gov.br. 
Tabela 1 - Sumário dos dados dos Estados brasileiros: período 1995-2003.

\begin{tabular}{|c|c|c|c|c|c|c|}
\hline Variável & & Média & Desvio Padrão & Mínimo & Máximo & Observações \\
\hline & Total & & 0,0265 & 0,0426 & 0,2456 & $\mathrm{~N}=243$ \\
\hline \multirow{2}{*}{ Txdes } & Entre & 0,1027 & 0,0202 & 0,0675 & 0,1483 & $\mathrm{n}=27$ \\
\hline & Dentro & & 0,0177 & 0,0212 & 0,2015 & $\mathrm{~T}=9$ \\
\hline \multirow{3}{*}{ Gini } & Total & & 0,0366 & 0,4246 & 0,6560 & $N=243$ \\
\hline & Entre & 0,5765 & 0,03032 & 0,4988 & 0,6149 & $\mathrm{n}=27$ \\
\hline & Dentro & & 0,0212 & 0,4713 & 0,6389 & $\mathrm{~T}=9$ \\
\hline \multirow{3}{*}{ W } & Total & & 0,4149 & 0,3586 & 2,4654 & $N=243$ \\
\hline & Entre & 0,9897 & 0,4163 & 0,4020 & 2,3593 & $\mathrm{n}=27$ \\
\hline & Dentro & & 0,0680 & 0,7888 & 1,2611 & $\mathrm{~T}=9$ \\
\hline \multirow{3}{*}{ Pibserv } & Total & & 0,1439 & 0,2650 & 1,1240 & $\mathrm{~N}=243$ \\
\hline & Entre & 0,5429 & 0,1419 & 0,3302 & 1,0064 & $n=27$ \\
\hline & Dentro & & 0,0353 & 0,4238 & 0,6604 & $\mathrm{~T}=9$ \\
\hline \multirow{3}{*}{ Pibind } & Total & & 0,1143 & 0,0621 & 0,6101 & $\mathrm{~N}=243$ \\
\hline & Entre & 0,2916 & 0,1114 & 0,0765 & 0,5511 & $n=27$ \\
\hline & Dentro & & 0,0326 & 0,2041 & 0,4204 & $\mathrm{~T}=9$ \\
\hline \multirow{3}{*}{ Pibagro } & Total & & 0,0709 & 0,0049 & 0,4517 & $\mathrm{~N}=243$ \\
\hline & Entre & 0,1046 & 0,0689 & 0,0058 & 0,2920 & $n=27$ \\
\hline & Dentro & & 0,0205 & 0,0514 & 0,2642 & $\mathrm{~T}=9$ \\
\hline \multirow{3}{*}{ Pibcome } & Total & & 0,0348 & 0,0217 & 0,2276 & $N=243$ \\
\hline & Entre & 0,0799 & 0,0337 & 0,0317 & 0,2107 & $n=27$ \\
\hline & Dentro & & 0,0108 & 0,0429 & 0,1520 & $\mathrm{~T}=9$ \\
\hline \multirow{3}{*}{$E$} & Total & & 0,6847 & 2,1592 & 6,4040 & $N=243$ \\
\hline & Entre & 4,1415 & 0,5019 & 2,9586 & 5,0844 & $\mathrm{n}=27$ \\
\hline & Dentro & & 0,4745 & 2,9141 & 6,0169 & $\mathrm{~T}=9$ \\
\hline
\end{tabular}

Fonte: PNAD; IPEADATA.

\subsubsection{Estimativas e testes econométricos}

Os testes a serem realizados, além daquele de especificação, serão os de autocorrelação e heterocedasticidade. Para maior compreensão, são apresentadas, de forma sucinta, as hipóteses do modelo e seus testes. A especificação do modelo econométrico para a equação (7) é a seguinte:

$$
\begin{aligned}
& U_{i t}=\beta U_{i, t-1}+\varphi W_{i, t}+\gamma X_{i, t}+\delta E_{i, t}+\tau E_{i, t}^{2}+\left(Z_{i, t}\right) \\
& Z_{i, t}=\alpha_{i}+\eta_{i, t}
\end{aligned}
$$

A variável $U_{i, t}$ representa a variável dependente com $i=1, \ldots, 27$ e $t=1, \ldots, 9 ; U_{t-1}, W, X, E$ e $E^{2}$ representam as variáveis independentes; $Z_{i, t}$ é o vetor que contempla os efeitos fixos dinâmicos. Assim, as hipóteses iniciais a serem estimadas sobre o modelo acima são as seguintes:

$$
\in\left[\alpha_{i}\right]=0
$$




$$
\begin{aligned}
& \in[\eta]=0 \\
& \in\left[\eta \eta^{\prime}\right]=\sigma_{\eta}^{2} I_{n T} \\
& \in\left[\alpha_{i} \alpha_{j}\right]=0, \text { para } \mathrm{i} \neq \mathrm{j} \\
& \in\left[\alpha_{i} \alpha_{i}\right]=\sigma_{\alpha}^{2} \\
& \in\left[\alpha_{i} \eta_{j t}\right]=0
\end{aligned}
$$

onde todos os valores esperados são condicionados pelas variáveis independentes. As hipóteses (10), (11) e (15) estabelecem as condições para que o modelo seja considerado de efeito randômico: a violação das mesmas indica a condição de modelo de efeitos fixos. O pressuposto essencial que distingue o modelo de efeitos randômicos do modelo de efeitos fixos é que o efeito específico de $\alpha_{\mathrm{i}}$, que é constante no tempo, não esteja correlacionado com as variáveis independentes. Desta forma, devemos estimar os dois modelos sob as condições de efeitos fixos e randômicos, para em seguida efetuar os testes de definição.

Na Tabela 2 a seguir, as variáveis salário médio e estrutura da economia dos Estados foram diferenciadas no tempo para atender a especificação da equação (7). ${ }^{11}$ Ou seja, a sua média foi extraída das observações no tempo. As variáveis Gini e escolaridade média foram utilizadas em diferenças e em níveis, ambas produzindo o mesmo resultado. Desta forma, optou-se por mantê-las em níveis para facilitar suas interpretações.

Os resultados das estimativas sob estas condições mostram que os modelos de efeito fixo (fe) e randômico (re) não apresentam os parâmetros como sendo significantes. A não significância dos parâmetros e o baixo poder de explicação podem estar associados aos problemas de heterocedasticidade e autocorrelação. Portanto, as informações de maior relevância nas regressões acima são: teste F na coluna (1), que indica que o modelo mais adequado é o de efeitos fixos, depois o alto valor da correlação entre os efeitos fixos e as variáveis independentes, correlação $\left(Z_{i}, \zeta_{i}\right) 67,9 \%$, o que ressalta a importância da correlação existente entre os efeitos fixos dos Estados e seus diferenciais persistentes no tempo.

Outra importante estatística é a $F$, que averigua se as taxas de desemprego de longo prazo dos Estados, $\alpha_{l}=0$, são iguais. De acordo com esta estatística, cada Estado tem sua própria taxa de desemprego, o que reforça a indicação de que as políticas de redução de desemprego devem ser diferenciadas. Estas diferenças persistentes e idiossincráticas das regiões (Estados) encontradas neste trabalho também estão presentes, implícita ou explicitamente, nos trabalhos de Corseuil et al. (1999), Oliveira e Cruz (2000); Oliveira e Carneiro (2001).

11 Foi adicionada a letra "d" de diferenciação nos nomes das variáveis. 
Tabela 2 - Modelos de efeito fixos e randômicos: dependente taxa de desemprego

\begin{tabular}{|c|c|c|}
\hline $\begin{array}{l}\text { Variáveis } \\
\text { (1) }\end{array}$ & $\begin{array}{l}\text { Fixo (fe) } \\
\text { (2) }\end{array}$ & $\begin{array}{c}\text { Randômico (re) } \\
\text { (3) }\end{array}$ \\
\hline Gini & $\begin{array}{l}-0,043 \\
(0,176)\end{array}$ & $\begin{array}{l}-0,058 \\
(0,058)\end{array}$ \\
\hline$D w^{(a)}$ & $\begin{array}{l}-0,007 \\
(0,706)\end{array}$ & $\begin{array}{l}0,016 \\
(0,064)\end{array}$ \\
\hline Dpibserv & $\begin{array}{l}-0,126 \\
(0,010)^{*}\end{array}$ & $\begin{array}{l}-0,054 \\
(0,216)\end{array}$ \\
\hline Dpibind & $\begin{array}{l}-0,028 \\
(0,616)\end{array}$ & $\begin{array}{l}-0,065 \\
(0,196)\end{array}$ \\
\hline Dpibagro & $\begin{array}{l}-0,159 \\
(0,010)^{*}\end{array}$ & $\begin{array}{l}-0,115 \\
(0,029)^{* *}\end{array}$ \\
\hline$E$ & $\begin{array}{c}0,042 \\
(0,032)^{* *}\end{array}$ & $\begin{array}{r}0,0498 \\
(0,008)^{*}\end{array}$ \\
\hline$E^{2}$ & $\begin{array}{l}-0,004 \\
(0,065)^{\text {**** }}\end{array}$ & $\begin{array}{l}-0,0049 \\
(0,023)^{* *}\end{array}$ \\
\hline Constante & $\begin{array}{c}0,025 \\
(0,607)\end{array}$ & $\begin{array}{l}-0,0867 \\
(0,060)^{* * * *}\end{array}$ \\
\hline $\begin{array}{c}R^{2} \text { - dentro } \\
\text { - entre } \\
\text { - total }\end{array}$ & $\begin{array}{l}0,153 \\
0,055 \\
0,004\end{array}$ & $\begin{array}{l}0,115 \\
0,322 \\
0,229\end{array}$ \\
\hline $\operatorname{corr}\left(\alpha_{i}+\eta_{i}\right)$ & $-0,6790$ & - \\
\hline $\begin{array}{l}\sigma_{\alpha+\eta} \\
\sigma_{\eta}\end{array}$ & $\begin{array}{l}0,0290 \\
0,0175\end{array}$ & $\begin{array}{l}0,0161 \\
0,0175\end{array}$ \\
\hline$F(26,209), \alpha_{i}=0$ & $\operatorname{Prob}(0,000)$ & - \\
\hline$N$ & 243 & 243 \\
\hline
\end{tabular}

Notas: (a) letra d junto à variável refere-se ao fato de se ter usado as séries em sua diferença. $\left(^{*}\right)$ indica nível de significância de 1\%. $\left(^{* *}\right)$ indica nível de significância de $5 \%,\left(^{* *}\right)$ indica nível de significância inferior a $10 \%$.

De acordo com Marston (1985), o desvio padrão da taxa de desemprego de equilíbrio, $\sigma_{\alpha}$, deve ser maior que o desvio padrão dos componentes do desemprego de desequilíbrio, $\sigma_{\eta}$. No entanto, neste modelo, foi encontrado um resultado contrário para a estimação do modelo de efeitos fixos. Quando estimado sob os pressupostos do modelo de efeitos randômicos, a disparidade em sentido oposto é ainda maior. Então, constata-se que o desvio padrão da taxa de desemprego de equilíbrio foi menor que o desvio padrão dos componentes do desemprego de desequilíbrio em ambas as estimações, isso reafirma a evidência dos efeitos fixos.

O teste de especificação proposto por Hausman (1978) verifica se os modelos de efeitos fixos e randômicos são idênticos. Caso sejam, a melhor especificação é a randômica, dada a condição de 
ortogonalidade entre os efeitos fixos e as variáveis independentes. O teste de Hausman obteve o seguinte resultado: teste Qui-Quadrado $x^{2}=12,51$, permitindo rejeitar a hipótese nula de igualdade dos coeficientes, sendo sua probabilidade de 0,050 (Prob $>x^{2}=0,050$ ). Esta probabilidade confirma o modelo de efeitos fixos como o mais adequado.

No entanto, este teste pode ser influenciado pela presença da autocorrelação. Os testes de Máxima Verossimilhança (MV) propostos por Breusch e Pagan (1980) e Baltagi e Li (1995) para efeitos randômicos, que considera a presença de autocorrelação, estão na Tabela 3 a seguir.

\section{Tabela 3 - Testes randômicos e correlação serial}

\begin{tabular}{lcc}
\hline $\begin{array}{l}\text { Testes } \\
\text { (1) }\end{array}$ & $\begin{array}{c}\text { Resultados } \\
(2)\end{array}$ & $\begin{array}{c}\text { Probabilidade } H_{0} \\
(3)\end{array}$ \\
Efeitos Randômicos & 120,29 & 0,000 \\
\hline MV: $\operatorname{Var}\left(u_{i}\right)=0$ & 56,06 & 0,000 \\
MVA: $\operatorname{Var}\left(u_{i}\right)=0$ & & \\
Correlação Serial & 85,73 & 0,000 \\
MV: $\rho=0$ & 21,50 & 0,000 \\
MVA: $\rho=0$ & & \\
Teste $\operatorname{Conjunto~}$ & 141,79 & 0,000 \\
MV: $\operatorname{Var}\left(u_{i}\right)=0$ e $\rho=0$ &
\end{tabular}

De acordo com os testes de efeitos randômicos, tanto o método de máxima verossimilhança sem correção para autocorrelação (MV) quanto o que considera correção para autocorrelação (MVA) informam que o modelo de efeitos fixos é o que deve ser considerado, dada a probabilidade de efeitos randômicos ser zero. Quanto à autocorrelação, a probabilidade de $\rho=0$ é zero, portanto, rejeita-se a hipótese nula de não autocorrelação tanto na versão $\mathrm{MV}$, quanto na MVA. O teste conjunto, que verifica a presença de efeito randômico e ausência de autocorrelação, também não se apresentou como sendo significante.

Outro teste específico para autocorrelação de painéis de dados foi sugerido Wooldridge (2002). Este teste foi desenvolvido por Drukker (2003), e o resultado do teste $\mathrm{F}(1,26)=30,40$ indica uma probabilidade zero de aceitação da não existência de autocorrelação de primeira ordem.

Efetua-se adicionalmente o teste de heterocedasticidade apresentado por Greene (2000). O resultado da estatística $x^{2}=321,49$ informa que a probabilidade de os painéis terem a mesma variância é zero, portanto, registra-se a presença de heterocedasticidade nos dados.

Este conjunto de testes indica que deve ser aplicado um modelo de efeitos fixos, levando em consideração (e/ou corrigindo) a presença de autocorrelação e heterocedasticidade. Para tanto, foram utilizados os métodos Prais-Winstem $(P-W)$, o qual estimará o modelo por Mínimo Quadrado Ordinário $(M Q O-P W)$, e também o método Mínimo Quadrado Generalizado Factível (FGLS), ambos levando em consideração os problemas de heterocedasticidade e autocorrelação - veja Tabela 4 a seguir. 
O resultado obtido por meio do método $P-W$, coluna (2) da Tabela 4, é deficiente na significância dos parâmetros. Portanto, os resultados anteriores persistem sob este método. As estimativas efetuadas por meio dos métodos FGLS, coluna (3) da Tabela 4, melhoraram o nível de significância das variáveis Gini, diferença de salários e escolaridade média. Neste caso, Estados que possuem péssima distribuição de renda são menos atrativos e, portanto, tendem a possuir menor taxa de desemprego, enquanto que a diferença salarial demonstra se um fator de atração é significante. A variável escolaridade média e o seu quadrado devem ser analisados conjuntamente. As significâncias das mesmas demonstram a existência de um comportamento não-linear que será explorado mais à frente. As variáveis que representam a estrutura da economia dos Estados não se apresentaram como sendo significantes. As razões prováveis são causalidade simultânea e omissão de variáveis. Assim, o modelo adequado que considera estes aspectos é o dinâmico, que apresentaremos a seguir.

Tabela 4 - Modelos P-W e FGLS: dependente taxa de desemprego

\begin{tabular}{|c|c|c|}
\hline $\begin{array}{l}\text { Variáveis } \\
\text { (1) }\end{array}$ & $\begin{array}{c}P-W \\
(2) \\
\end{array}$ & $\begin{array}{c}F G L S \\
(3)\end{array}$ \\
\hline Gini & $\begin{array}{l}-0,069 \\
(0,112)\end{array}$ & $\begin{array}{c}-0,043 \\
(0,001)\end{array}$ \\
\hline$D w^{(a)}$ & $\begin{array}{c}0,014 \\
(0,002)^{*}\end{array}$ & $\begin{array}{c}0,015 \\
(0,000)\end{array}$ \\
\hline Dpibserv & $\begin{array}{c}0,007 \\
(0,841)\end{array}$ & $\begin{array}{c}0,026 \\
(0,482)\end{array}$ \\
\hline Dpibind & $\begin{array}{l}-0,051 \\
(0,266)\end{array}$ & $\begin{array}{l}-0,017 \\
(0,696)\end{array}$ \\
\hline Dpibagro & $\begin{array}{l}-0,048 \\
(0,248)\end{array}$ & $\begin{array}{l}-0,006 \\
(0,880)\end{array}$ \\
\hline$E$ & $\begin{array}{c}0,057 \\
(0,006)^{*}\end{array}$ & $\begin{array}{c}0,052 \\
(0,000)\end{array}$ \\
\hline$E^{2}$ & $\begin{array}{l}-0,006 \\
(0,031)^{* *}\end{array}$ & $\begin{array}{c}-0,005 \\
(0,000)\end{array}$ \\
\hline Constante & $\begin{array}{l}-0,096 \\
(0,019)^{*}\end{array}$ & $\begin{array}{l}-0,014 \\
(0,418)\end{array}$ \\
\hline $\begin{array}{c}R^{2} \text { - dentro } \\
\text { - entre } \\
\text { - total }\end{array}$ & $\begin{array}{c}- \\
- \\
0,280\end{array}$ & $\begin{array}{l}- \\
- \\
-\end{array}$ \\
\hline $\operatorname{corr}\left(\alpha_{i}+\eta_{i}\right)$ & - & - \\
\hline $\begin{array}{l}\sigma_{\alpha+\eta} \\
\sigma_{\eta} \\
\end{array}$ & - & - \\
\hline$F(26,209), \alpha_{i}=0$ & - & - \\
\hline$N$ & 243 & 243 \\
\hline
\end{tabular}

Notas: (a) letra d junto à variável refere-se ao fato de se ter usado as séries em sua diferença. $\left(^{*}\right)$ indica nível de significância de 1\%. $\left(^{* *}\right)$ indica nível de ignificância de $5 \%,(* *)$ indica nível de significância inferior a $10 \%$. 


\subsubsection{Aplicação do modelo econométrico dinâmico}

As estimativas por meio do modelo econométrico dinâmico (MED) visam obter os coeficientes que consideram o efeito feedback da própria variável dependente (desemprego), como sugere Arellano e Bond (1991). O modelo proposto é uma especificação da equação (7), pois considera os efeitos da própria taxa de desemprego passada $\left(U_{t-1}\right)$ sobre o desemprego atual $\left(U_{t}\right)$, em nível e em diferença; portanto, um sistema de equações.

$$
U_{i t}=\beta U_{i, t-1}+\varphi W_{i, t}+\gamma X_{i, t}+\delta E_{i, t}+\tau E_{i, t}^{2}+\left(Z_{i, t}\right)
$$

$$
\begin{aligned}
U_{i, t}-U_{i, t-1}= & \beta\left(U_{i, t-1}-U_{i, t-2}\right)+\varphi\left(W_{i, t}-W_{i, t-1}\right)+\gamma\left(X_{i, t}-X_{i, t-1}\right)+\delta\left(E_{i, t}-E_{i, t-1}\right) \\
& +\tau\left(E_{i, t}^{2}-E_{i, t-1}^{2}\right)+\left(Z_{i, t}-Z_{i, t-1}\right)
\end{aligned}
$$

Ao estimar a equação usando as primeiras diferenças das variáveis explicativas, serão removidos os efeitos fixos, ficando com uma equação que pode ser estimada usando variáveis instrumentais. Assim, os instrumentos da equação em níveis são as variáveis defasadas em diferenças e, para a equação em diferença, são as defasadas em níveis, segundo Blundell e Bond (1998). Para que haja ortogonalidade, a seguinte condição se faz necessária:

$$
\in\left(U_{i, t-s} \Delta \eta_{i t}\right)=0 \text { para todo } t=3, \ldots, \mathrm{T} \text { e } s \geq 2
$$

onde $\Delta \eta_{i t}=\eta_{i t}-\eta_{i, t-1}$. Isto depende de se assumir a ausência de correlação serial dos distúrbios $\eta_{i t}$ variando com o tempo juntamente com a seguinte restrição: $\in\left(U_{i 1} \eta_{i 1}\right)=0$ para $i=1, \ldots, N$ e $t=2, \ldots, T$.

Para a estimativa na forma de sistemas, o Método de Momentos Generalizados (GMM) deve ser utilizado e também as seguintes condições iniciais dos momentos devem ser obedecidas:

$$
\begin{aligned}
& \in\left(\eta_{i t} \Delta U_{i, t-1}\right)=0 \text { para todo } t=4,5, \ldots T \\
& \in\left(\eta_{i 3} \Delta U_{i 2}\right)=0
\end{aligned}
$$

Quanto à variável dependente defasada, pressupõe-se que se o desemprego no período anterior reflete todas as variáveis que o influenciaram, então, ao considerarmos como variável explicativa, estas são consideradas de forma indireta (Dias et al., 2005). Quanto aos efeitos contemporâneos sobre a taxa de desemprego, estes devem ser, então, oriundos das demais variáveis explicativas, propostas no modelo.

Quando estimada na forma de diferença, a variável gini é utilizada como variável predeterminada e endógena, com apenas um lag. As diferenças das demais variáveis explicativas também serão estimadas de forma predeterminada e usando defasagens de três ou mais períodos como instrumentos. Para a estimativa dos resultados contidos na coluna (2) da tabela a seguir, foram usados quatro períodos de defasagem para a variável taxa de desemprego (txdes), portanto uma especificação dinâmica. No que concerne aos resultados das colunas (3), (4) e (5), a particularidade é 
o uso de diferentes momentos para cada variável explicativa. Diferentemente das demais variáveis, a diferença da taxa de desemprego (dtxdes) e a diferença da variável salário $(d w)$ entram com três períodos de defasagens entre os termos independentes, enquanto as variáveis que representam escolaridade média dos desempregados $\left(E\right.$ e $\left.E^{2}\right)$ estão todas em nível. Esta foi a melhor especificação usando o critério de seleção de minimização da função objetivo.

As equações foram estimadas, primeiramente, sob a condição de que a variável taxa de desemprego em nível precede o próprio desemprego contemporâneo em quatro anos, e as variáveis escolaridade e escolaridade ao quadrado são precedidas por elas mesmas em três anos (instrumentos). $\mathrm{Na}$ Tabela 5, encontram-se os resultados do modelo estimado com quatro defasagens, funcionando como instrumentos e, depois, o mesmo modelo com sistema GMM e variáveis predeterminadas como instrumentos.

Quanto aos resultados da coluna 2 da Tabela 5, seguindo os procedimentos sugeridos por Arellando e Bond (1991), em que somente as diferenças defasadas são usadas como instrumentos, apresentam-se muito satisfatórios para escolaridade média e escolaridade média ao quadrado. Estimou-se o modelo com quatro defasagens e todas apresentaram estatísticas significativas. Quanto às demais variáveis, com exceção das variáveis salário e PIB do setor serviços, as demais são significativas e coerentes com a teoria.

Os resultados das colunas (3), (4) e (5), da Tabela 5 a seguir, apresentam resultados mais consistentes, dada a metodologia de sistema de equações GMM proposta por Blundel e Bond (1998). O sinal encontrado para a taxa de desemprego ocorrida em $t$-3, como pode ser visto nestas três últimas estimações, é contrário àquele encontrado na coluna (2). Enquanto na primeira estimação uma alta taxa de desemprego no passado levava a uma menor taxa de desemprego no período contemporâneo, nas estimações das colunas (3), (4) e (5), as altas taxas de desemprego no passado ( $t$-3) explicam boa parte da taxa de desemprego do presente. Assim, estes últimos resultados são mais consistentes do que o resultado da primeira estimação, coluna (2).

O coeficiente Gini, que representa a estrutura da distribuição de renda dos Estados, retrata o resultado da distribuição do pós-trabalho nos Estados. O sinal do coeficiente Gini contemporâneo é negativo e positivo defasado. Este resultado negativo indica que os Estados que possuem má distribuição de renda são menos atrativos. Ou, em outras palavras, quanto mais equitativa for a distribuição de renda nos Estados, maior será a taxa de desemprego.

Com relação aos salários (w), espera-se que, quanto maior forem os níveis (valores) desta variável, esta terá um efeito positivo (aumentará) sobre a taxa de desemprego dos Estados. Neste caso, como pode ser visto nas colunas (3), (4) e (5) da tabela anterior, os resultados estão de acordo com os pressupostos iniciais no que se refere aos sinais dos parâmetros. Os parâmetros encontrados para a curva de salários, apesar de serem bastante pequenos, são estatisticamente aceitáveis e estão de acordo com os pressupostos teóricos de que regiões onde prevalece o maior salário médio tendem a possuir maior taxa de desemprego, devido a sua atratividade. 
Tabela 5 - Modelo Econométrico Dinâmico (MED) explicando a taxa de desemprego dos Estados

\begin{tabular}{|c|c|c|c|c|}
\hline $\begin{array}{l}\text { Variáveis } \\
\text { (1) }\end{array}$ & $\begin{array}{l}\text { MED } \\
(2)\end{array}$ & $\begin{array}{l}\text { MED } \\
(3)\end{array}$ & $\begin{array}{l}\text { MED } \\
(4)\end{array}$ & $\begin{array}{l}\text { MED } \\
(5)\end{array}$ \\
\hline & DIFERENÇA & & SISTEMA - GMM & \\
\hline $\begin{array}{l}\text { dtxdes } \\
\text { L2 } \\
\text { L3 } \\
L 4\end{array}$ & $\begin{array}{c}-0,6241^{*} \\
(0,000) \\
-0,4450^{*} \\
(0,000) \\
-0,0984^{*} \\
(0,007) \\
-0,1532^{*} \\
(0,000)\end{array}$ & $\begin{array}{l}- \\
- \\
0,6023^{*} \\
(0,000) \\
-\end{array}$ & $\begin{array}{l}- \\
- \\
0,6014^{*} \\
(0,000) \\
-\end{array}$ & $\begin{array}{c}- \\
- \\
0,6073^{*} \\
(0,000) \\
-\end{array}$ \\
\hline $\begin{array}{l}\text { Gini } \\
\text { L1 }\end{array}$ & $\begin{array}{l}-0,0234^{* * *} \\
(0,070) \\
-\end{array}$ & $\begin{array}{c}-0,0189 \\
(0,618) \\
0,0505 \\
(0,097)\end{array}$ & $\begin{array}{c}-0,0189 \\
(0,613) \\
0,0509 \\
(0,095)\end{array}$ & $\begin{array}{c}-0,129 \\
(0,694) \\
0,0546 \\
(0,083)\end{array}$ \\
\hline $\begin{array}{l}d w \\
L 3\end{array}$ & $\begin{array}{l}-0,0029 \\
(0,726)\end{array}$ & $\begin{array}{l}- \\
0,0106^{\star * *} \\
(0,092)\end{array}$ & $\begin{array}{l}- \\
0,0107^{\star \star *} \\
(0,091)\end{array}$ & $\begin{array}{c}- \\
0,0121^{*} \\
(0,057)\end{array}$ \\
\hline $\begin{array}{l}\text { dPibagro } \\
\text { L1 }\end{array}$ & $\begin{array}{c}-0,0496 \\
(0,147) \\
-\end{array}$ & $\begin{array}{c}-0,1077^{*} \\
(0,003) \\
0,1040^{*} \\
(0,008)\end{array}$ & $\begin{array}{c}-0,1089^{*} \\
(0,002) \\
0,0968^{* *} \\
(0,018)\end{array}$ & $\begin{array}{c}-0,1101^{*} \\
(0,001) \\
0,1023^{*} \\
(0,009)\end{array}$ \\
\hline dpibserv & $\begin{array}{l}-0,0028 \\
(0,926)\end{array}$ & $\begin{array}{c}0,0085 \\
(0,577)\end{array}$ & - & - \\
\hline dpibind & -- & - & $\begin{array}{l}-0,0105 \\
(0,501)\end{array}$ & - \\
\hline dpibcome & -- & - & - & $\begin{array}{c}0,0145 \\
(0,724)\end{array}$ \\
\hline$E$ & $\begin{array}{c}0,0507^{*} \\
(0,017)\end{array}$ & $\begin{array}{c}0,0544^{*} \\
(0,033)\end{array}$ & $\begin{array}{l}0,0539^{* *} \\
(0,030)\end{array}$ & $\begin{array}{c}0,0510^{* *} \\
(0,031)\end{array}$ \\
\hline$E^{2}$ & $\begin{array}{l}-0,0075^{*} \\
(0,001)\end{array}$ & $\begin{array}{l}-0,0066^{* *} \\
(0,020)\end{array}$ & $\begin{array}{l}-0,0065^{* *} \\
(0,018)\end{array}$ & $\begin{array}{l}-0,0062^{* *} \\
(0,024)\end{array}$ \\
\hline Constante & $\begin{array}{c}0,0075^{*} \\
(0,000)\end{array}$ & $\begin{array}{l}-0,0158 \\
(0,732)\end{array}$ & $\begin{array}{l}-0,0151 \\
(0,745)\end{array}$ & $\begin{array}{l}-0,0136 \\
(0,786)\end{array}$ \\
\hline$F(9,26)=20,69$ & - & 0,000 & - & - \\
\hline$F(9,26)=20,95$ & - & - & 0,000 & - \\
\hline$F(9,26)=25,37$ & - & - & - & 0,000 \\
\hline Sargan:Prob $\left(X^{2}\right)$ & 0,833 & - & - & \\
\hline Teste Hansen & - & 0,990 & 0,990 & 0,990 \\
\hline$A R 1$ & 0,055 & 0,172 & 0,172 & 0,175 \\
\hline$A R 2$ & 0,415 & 0,785 & 0,770 & 0,823 \\
\hline
\end{tabular}

Nota: (1) estimativas dinâmicas utilizando estimador GMM de primeira diferença. (*) indica nível de significância inferior a 1\%. $\left(^{* *}\right)$ indica nível de significância inferior a 5\%. $\left(^{* *}\right)$ indica nível de significância inferior a $10 \%$. 
No que se refere à estrutura da economia, representada pelas variáveis PIB dos setores, verificou-se que o sinal é realmente negativo; quando positivo, não apresentou níveis de significância aceitáveis. Isso implica que políticas de estímulo ao setor agrícola, por exemplo, devem contribuir para a diminuição das taxas de desemprego nos Estados. ${ }^{12}$ Uma explicação plausível é que este setor (agrícola) exerce importante papel na demanda por mão-de-obra pouco qualificada.

Considerando a relevância dos resultados quando foram usadas as defasagens, pode-se confirmar a hipótese de que os efeitos das políticas de longo prazo em cada Estado são importantes, uma vez que políticas realizadas em períodos anteriores contribuem para diminuir a taxa de desemprego no período corrente.

A significância da variável escolaridade média dos desempregados demonstra a sua influência direta sobre a taxa de desemprego. Este resultado está coerente com os pressupostos teóricos de não-linearidade, conforme Kueguer e Lindahl (1999) e Dias et al. (2005). A função quadrática ${ }^{13}$ da escolaridade média dos desempregados $(E)$ para explicar a taxa de desemprego, pode ser especificada da seguinte maneira: $U=\beta_{1} \mathrm{E}-\beta_{2} \mathrm{E}^{2}$, onde $E^{2}$ é escolaridade média dos desempregados ao quadrado.

Por meio dos valores estimados na Tabela 4 e coluna (4) chega-se à seguinte equação:

$$
U=0,0539 \mathrm{E}-0,0065 \mathrm{E}^{2}
$$

Calculando a derivada parcial da equação (21) e igualando a zero,

$$
\frac{\partial U}{\partial \mathrm{E}}=0,0539-0,0065(2) \mathrm{E}=0
$$

Verifica-se que a taxa ótima de $E^{*}$ é 4,15 anos de estudo. Portanto, os Estados em que os níveis de escolaridade média dos desempregados estão abaixo desse nível devem privilegiar suas políticas educacionais como forma de diminuir suas taxas de desemprego. Assim, qualquer valor de $E>E^{*}$ contribuirá para aumentar o nível de empregabilidade dos trabalhadores. Para melhor compreensão dessa relação entre desemprego $(U)$ e escolaridade $(E)$, o comportamento da função quadrática especificada para a taxa de desemprego pode ser visualizado na Figura 1 a seguir.

12 Dada a heterogeneidade das unidades da Federação brasileira, as políticas de estímulos setoriais devem ser bastante específicas para alcançar seus objetivos. Sabe-se que determinados Estados (regiões) podem ser predominantemente agrícolas, enquanto outras são mais industrializadas.

13 A adição de um termo quadrático à equação linear tem como objetivo capturar de forma simples o efeito dos rendimentos decrescentes. Desta forma, como pode ser visto na equação (21), a partir do momento que $E>E$, um aumento em $E$ diminuirá a taxa de desemprego $(U)$, dado o pressuposto do termo quadrático. Em termos gráficos, quando a $E$ ultrapassa seu ponto crítico, a inclinação da função diminuirá para maiores níveis de escolaridade. 
Figura 1 - Efeito da escolaridade média dos desempregados sobre a taxa de desemprego

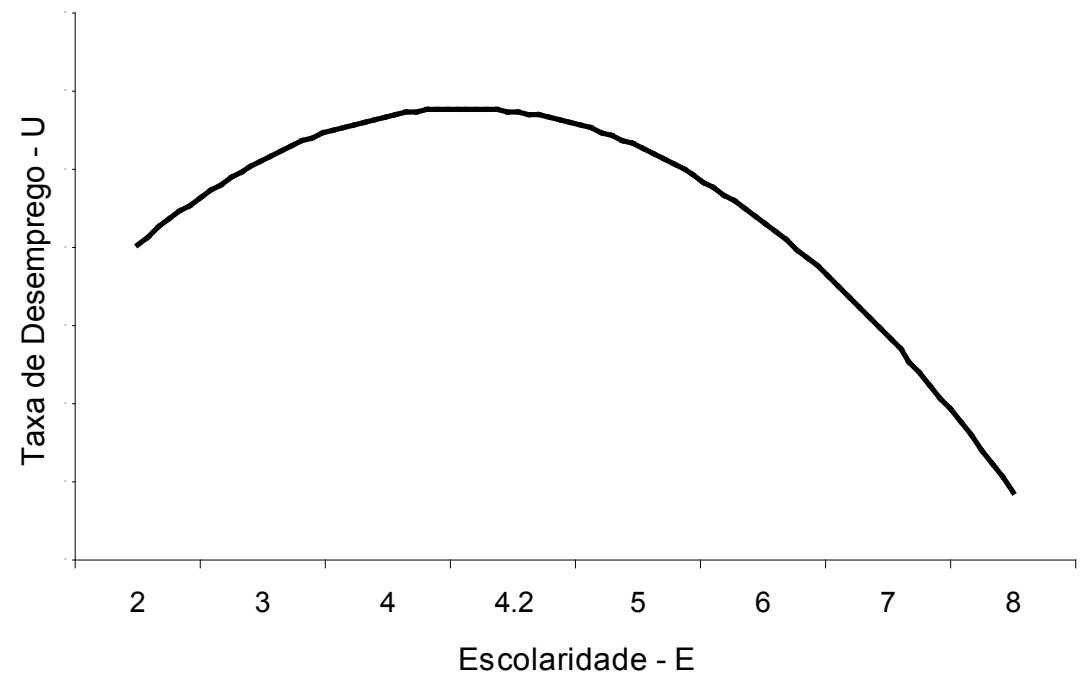

A Figura 1 mostra um efeito marginal decrescente da evolução da taxa de desemprego quando aumenta a escolaridade média dos desempregados. Assim, quando o valor de $E$ (escolaridade média dos desempregados) é baixo, tem-se um aumento na taxa de desemprego. Porém, se aumentar $E$ pela mesma quantidade constantemente, esse aumento no nível de E diminuirá a taxa de desemprego.

Considerando que as estimativas em primeira diferença apresentaram-se significativas com defasagens de quatro anos, enquanto que as estimações por meio de sistemas GMM foram significantes com defasagens de 3 anos, pode-se inferir que os investimentos em educação, que aumentam o nível médio de escolaridade, levam, em média, de três a quatro anos para reduzir a taxa de desemprego dos Estados no Brasil, não apresentando um retorno linear.

Quanto aos instrumentos, a condição de validade dos mesmos é inferida pelos testes estatísticos de Sargan e Hansen. A probabilidade de os instrumentos serem ortogonais é de $83 \%$ e $99 \%$, respectivamente. A validade dos resultados acima está subordinada à condição de auto-regressividade. As estimativas são válidas, neste método, sob a condição de não auto-regressão de segunda ordem, (AR2), podendo admitir a de primeira ordem, (AR1). As estatísticas confirmam a ausência de AR1 e AR2. Ou seja, no conjunto, os resultados podem confirmar a hipótese de que, controlando as condições iniciais da economia (variáveis não-observáveis) e as demais variáveis, a baixa escolaridade média dos desempregados é uma das causas de certos Estados terem taxas de desemprego mais elevadas.

De acordo com os resultados empíricos obtidos, comprova-se a hipótese de existência de um nível médio de escolaridade, a partir da qual a taxa de desemprego declina continuamente. Quando analisada sob esta ótica, foi constatado que as únicas regiões que possuem níveis médios de escolaridade acima de $E^{*}$ (ponto ótimo da escolaridade) foram: região Sudeste e região Sul; portanto as demais regiões que estão abaixo desse nível de escolaridade devem privilegiar suas políticas educacionais como forma de obterem menores taxas de desemprego no longo prazo. 


\section{CONSIDERAÇÕES FINAIS}

Neste trabalho foi desenvolvido um modelo que considera a importância dos chamados efeitos fixos dos Estados sobre a taxa de desemprego dos mesmos. Este modelo teve como característica principal considerar o papel da escolaridade média dos desempregados.

As estimativas dinâmicas em painéis de dados visando explicar a taxa de desemprego dos Estados demonstraram suporte ao modelo estendido de Marston (1985). A relação entre escolaridade e taxa de desemprego demonstrou ser não-linear, possuindo o formato de U invertido. A escolaridade média dos desempregados nos Estados apresentou um efeito quadrático interessante. Para níveis baixos de escolaridade média dos desempregados ( $\mathrm{E}<4,15$ anos de estudos), o efeito sobre a taxa de desemprego é positivo, o que leva a um aumento na taxa de desemprego; no entanto, para escolaridade média acima deste valor ( $\mathrm{E}>4,15$ anos) temos um efeito negativo sobre a taxa de desemprego com uma defasagem de três anos.

Considerando que a escolaridade média dos desempregados exerce importante papel, as políticas de combate ao desemprego devem ter como foco a continuidade da educação dos que estejam desempregados. E ainda considerar que, de acordo com o modelo, os investimentos em educação que aumentam os níveis médios de escolaridade dos desempregados levam em média três anos para reduzir a taxa de desemprego dos Estados no Brasil, sendo ainda seu efeito não linear.

\section{REFERÊNCIAS BIBLIOGRÁFICAS}

AHN, S. C.; SCHMIDT, P. Eficient estimation of models for dynamic panel data. Journal of Econometrics, v. 68, p. $5-27,1995$.

ARELLANO, M.; BOND, S. R. Some tests of specification for panel data: Monte Carlo evidence and an application to employment equations. Review of Economic Studies, n. 58, p. 277-297, 1991.

ARELLANO, M.; BOVER, O. another look at the instrumental variable estimation of error-components models. Journal of Econometrics, v. 68, p. 29-51, 1995.

BALTAGI, B. H.; LI, Q. Testing AR(1) Against MA(1) disturbances in an error component model. Journal of Econométrics. v. 68, p.133-151, 1995.

BLUNDELL, R.; BOND, S. Initial conditions and moment restrictions in dynamic panel data models. Journal of Econometrics, v. 87, p. 115-143, 1998.

BREUSCH, T.; PAGAN, A. The LM and its application to model specification in econometrics. Review of Economic Studies, n. 47, p. 239-254, 1980.

CAMARGO, J. M.; REIS, M. C. Desemprego: o custo da desinformação. Revista Brasileira de Economia, v. 59, n. 3, p. 381-425, 2005.

CHAHAD, J. P. Z. Tendências recentes no mercado de trabalho: pesquisa de emprego e desemprego. São Paulo Perspectiva, v. 17, n. 3-4, 2003.

CORSEUIL, C. H.; GONZAGA, G.; ISSLER, J. V. Desemprego regional no Brasil: uma abordagem empírica. Revista de Economia Aplicada, v. 3, n. 3, p. 407-435, 1999.

DIAS, J.; DIAS, M. H. A.; LIMA, F. F. Crescimento econômico e nível de escolaridade: teoria e estimativas dinâmicas em painel de dados. Anais do XXXIII Encontro Nacional de Economia, Natal, RN, 2005.

DRUKKER, D. M. Testing for serial correlation in linear panel-data models. Stata Journal, v. 3, n. 2, p. 168-177, 2003. 
GIOVANNETTI, B.; MENEZES-FILHO, N. A. liberalização comercial e demanda por trabalho qualificado no Brasil. In: Anais do XXXIII Encontro Nacional de Economia, Natal-RN, 2005.

GONZAGA, G.; TERRA, M. C.; MENEZES-FILHO, N. The liberalization and the evolution of skill earnings differencials in Brazil. PUC-Rio, 2002. (Texto para Discussão n. 463).

GREENE, W. H. Econometrics analysis. New Jersey: Prentice Hal, 2000.

HAUSMAN, J. A. Especification tests in Econometrics. Econometrica, n. 46, p. 1251-1272, 1978.

KRUEGER, A, B.; LINDAHL, M. Education for growth: why and for whom? The Journal of Economic Literature. v. 39, n. 4, p. 1101-1136, 1999.

MARSTON, S. Two views of the geographic distribution of unemployment. The Quartely Journal of Economics, v. 100, n. 1, p. 57-79, 1985.

OLIVEIRA, C. W.; CRUZ, B. O. Desigualdades regionais e elasticidade de longo prazo do emprego nos Estados do Nordeste com relação ao emprego nacional. IPEA, 2000. (Texto para Discussão n. 704).

OLIVEIRA, C. W.; FRANCISCO, G. C. Flutuações de longo prazo do emprego no Brasil: uma análise alternativa de co-integração. Revista Brasileira de Economia, v. 55, n. 4, p. 493-512, 2001.

PISSARIDES, C.A.; McMASTER, I. Regional migration, wages and unemployment: empirical evidence and implication for policy. Oxford Economic Papers, n. 42, p. 812-831, 1990.

REIS, M. C. Mudanças no desemprego e nos rendimentos por nível de qualificação durante a década de noventa. Anais do XXXII Encontro Nacional de Economia, João Pessoa, PB, 2004.

TAYLOR, J. R. Staggered wage setting in a macro model. American Economic Review, v. 69, n. 2, 1979.

WOOLDRIDGE, J. M. Econometric analysis of cross section and panel data. Cambridge: The MIT Press, 2002. 